\title{
RENAISSANCE PHAGE
}

In today's culture of spin, 'renaissance' is a term that is often applied undeservingly to particular

areas of science. In the case of current phage research, however, its use is easily justified.

It is almost 90 years since phages were first isolated. Thanks largely to the efforts of Felix d'Herrelle, phage therapy was initially regarded with great promise. The advent of antibiotics in the 1930s was instrumental in the widespread decline in interest in this 'alternative' therapy however and, by the late 1940s, phage therapy was largely abandoned, at least in the West.

Fortunately, although interest in phage therapy declined, the fundamental mechanisms of phage genetics and biology remained at the forefront of biology. Phage became the main model system in molecular biology and remained so until sufficient basic knowledge allowed researchers to move on to other model organisms and interest in phage per se began to wane, around the early 1970s. There is widespread agreement however that phage research is now experiencing a renaissance, with the recognition of the importance of phage in bacterial pathogenesis and evolution and the revival of interest in phage therapy to name just a few areas.

An examination of the phage biology literature from the past 2-3 years reveals so many papers that it would have been genuinely difficult to choose highlights for this short editorial, had it not been for the 1st November 2004 issue of the ASM's Journal of Bacteriology. This issue devotes 36 pages to the complete genome sequence of bacteriophage $\mathrm{P} 1$, and an excellent accompanying commentary by Michael Yarmolinsky, one of the authors of the genome paper, puts the paper in context. As Yarmolinsky acknowledges, by publishing such an "extravagantly long" report, the Journal of Bacteriology "has shown what appears to be extraordinary indulgence". However, considering the contribution of P1 to molecular biology - both in the early days, as a general transducing phage mediating exchange of genetic material - and in the modern era, with the widespread use of the P1-derived Cre-lox system - one can only conclude that the attention is justified.

The publication of the 94,800 -bp sequence of $\mathrm{P} 1$ does, however, raise some interesting questions. The first complete genome sequence of an organism was published by Sanger and colleagues in 1977 and it was a phage genome
— all 5,375 nucleotides of $\Phi$ X174. With the advent of high-throughput, low-cost sequencing in the 1990s, it seems reasonable to assume that the number of complete phage genome sequences would have increased exponentially since that first report. Instead, although almost 200 complete bacterial genome sequences have now been deposited in GenBank, at the time of going to press only 237 complete phage genome sequences were available.

So why has it taken until 2004 to completely sequence a genome that, at $<100 \mathrm{~kb}$, a top-of-the-range automated sequencer could eat for breakfast? Well, in a way, that is exactly the problem - to a large extent, phage genomics has been 'out-scaled' by developments in genomics technology and, until recently, the large genome sequencing consortia showed little interest in these microbial small fry. Almost all of the phage genome sequences completed so far are the result of work by individual researchers or small research groups and, because many of the commercially available gene prediction programs require sequence lengths of at least $100 \mathrm{~kb}$, annotation can be a painstaking, laborious process. Moreover, because of their extraordinary diversity, it is not uncommon for more than $50 \%$ of the genes in a phage genome to lack any known homologues.

However, those phage researchers who stuck it out through the lean years are known for their 'infectious' enthusiasm, and the message seems to be getting through. The rate of completion of complete phage genome sequences has been increasing rapidly and sufficient data are available for some excellent comparative genomics studies. Moreover, funding bodies like the National Science Foundation have become involved in collaborative phage sequencing efforts and TIGR were involved in determining the sequence of the T4-like marine vibriophage KVP40. Additionally, this year saw the largest 'pure' phage meeting - 'The New Phage Biology', held in Key Biscayne, Florida - for many years and, thanks to Ry Young's sterling work, the meeting was a resounding success and will hopefully be a regular fixture in the ASM Meetings calendar. So, the future looks bright for those who believe "all the world's a phage". 